\title{
Folic acid during pregnancy alters rat hepatic phosphoenolpyruvate carboxykinase promoter methylation contingent on sex
}

\author{
S. P. Hoile ${ }^{1}$, K. A. Lillycrop ${ }^{2}$, N. A. Thomas ${ }^{1}$, M. A. Hanson ${ }^{1}$ and G. C. Burdge ${ }^{1}$ \\ ${ }^{1}$ University of Southampton School of Medicine and ${ }^{2}$ University of Southampton Faculty of Natural Sciences, Southampton, \\ SO16 6YD, UK
}

Women who intend to become pregnant or who are in their first trimester are recommended to supplement their diet with folic acid in order to reduce risk of neural tube defects in their fetus ${ }^{(1)}$. However, studies in rats show that folic acid supplementation during pregnancy induces long-term changes in gene expression in other tissues including the liver ${ }^{(2)}$. In order to investigate the mechanism underlying such effects we measured the methylation status of individual $\mathrm{CpG}$ dinucleotides in the hepatic phosphoenolpyruvate carboxykinase (PEPCK) promoter in the adult offspring of rats fed either an adequate amount of folic acid (FA) or a diet with a comparable increment in FA content to that recommended to pregnant women diet during pregnancy (FA supplemented (FS)).

The study was carried out in accordance with the Home Office Animals (Scientific Procedures) Act (1986). Virgin female Wistar rats ( $n 5$ per dietary group) were fed either a FA ( $1 \mathrm{mg} / \mathrm{kg}$ folic acid) or FS $(5 \mathrm{mg} / \mathrm{kg}$ feed folic acid) diet throughout pregnancy and AIN93G during lactation. Offspring were weaned on PN day 28 onto AIN93M diet and killed by on day 90. Offspring ( $n 5$ males or females per group) were fasted for $12 \mathrm{~h}$ prior to sample collection. Hepatic PEPCK mRNA expression was measured by real-time RTPCR ${ }^{(2)}$. PEPCK promoter methylation was determined by pyrosequencing ${ }^{(3)}$. Statistical analysis was by a general linear model (GLM) with sex and the FA content of maternal diet (F) as fixed factors. The numerical designation of CpGs refers to their location (bp) from the transcription start site.

\begin{tabular}{|c|c|c|c|c|c|c|c|c|c|c|c|c|c|c|}
\hline & \multirow{2}{*}{\multicolumn{2}{|c|}{$\begin{array}{l}\text { Expression } \\
(\log \Delta \Delta \mathrm{ct})\end{array}$}} & \multicolumn{12}{|c|}{ CpG methylation $(\%)$} \\
\hline & & & \multicolumn{2}{|c|}{-606} & \multicolumn{2}{|c|}{-508} & \multicolumn{2}{|c|}{-440} & \multicolumn{2}{|c|}{-129} & \multicolumn{2}{|c|}{-90} & \multicolumn{2}{|c|}{-81} \\
\hline & Mean & $\overline{\mathrm{SD}}$ & Mean & $\overline{\mathrm{SD}}$ & Mean & $\overline{\mathrm{SD}}$ & Mean & $\overline{\mathrm{SD}}$ & Mean & $\overline{\mathrm{SD}}$ & Mean & $\overline{\mathrm{SD}}$ & Mean & $\mathrm{SD}$ \\
\hline Male FA & $0.48^{\mathrm{a}}$ & 0.3 & $29.0^{\mathrm{a}}$ & 1.7 & $26.5^{\mathrm{a}}$ & 3.1 & $30.7^{\mathrm{ac}}$ & 2.2 & 20.3 & 4.6 & 17.7 & 3.4 & 16.5 & 1.9 \\
\hline Male FS & $0.5^{\mathrm{a}}$ & 0.4 & $29.4^{\mathrm{a}}$ & 2.5 & $28.4^{\mathrm{a}}$ & 1.5 & $40.4^{\mathrm{b}}$ & 2.9 & 18.3 & 2.3 & 15.3 & 1.8 & 15.0 & 1.5 \\
\hline Female FA & $0.85^{\mathrm{b}}$ & 0.3 & $42.8^{\mathrm{b}}$ & 8.3 & $32.0^{\mathrm{a}}$ & 6.6 & $27.5^{\mathrm{a}}$ & 1.1 & 19.0 & 1.4 & 14.7 & 1.0 & 13.7 & 1.4 \\
\hline Female FS & $1.02^{\mathrm{b}}$ & 0.3 & $47.3^{\mathrm{b}}$ & \multicolumn{4}{|r|}{ GLM $(P)$} & 0.9 & 19.2 & 1.9 & 15.4 & 1.5 & 16.2 & 1.9 \\
\hline Sex & \multicolumn{2}{|c|}{0.007} & & & $<0$ & & $<0$. & & \multicolumn{2}{|c|}{ NS } & \multicolumn{2}{|c|}{ NS } & \multicolumn{2}{|c|}{ NS } \\
\hline $\mathrm{F}$ & \multicolumn{2}{|c|}{ NS } & \multicolumn{2}{|c|}{ NS } & \multicolumn{2}{|c|}{0.007} & \multicolumn{2}{|c|}{$<0.0001$} & \multicolumn{2}{|c|}{ NS } & \multicolumn{2}{|c|}{ NS } & \multicolumn{2}{|c|}{ NS } \\
\hline $\operatorname{Sex} * \mathrm{~F}$ & \multicolumn{2}{|c|}{ NS } & \multicolumn{2}{|c|}{ NS } & \multicolumn{2}{|c|}{0.049} & \multicolumn{2}{|c|}{0.125} & \multicolumn{2}{|c|}{ NS } & \multicolumn{2}{|c|}{ NS } & \multicolumn{2}{|c|}{0.014} \\
\hline
\end{tabular}

Different superscripts indicate significantly different values $(P<0.05)$ by Bonferroni's post-hoc test, NS, not significant.

PEPCK mRNA expression was higher in females than males, but was not altered by maternal FA intake. The methylation of specific CpG dinucleotides differed with both sex and maternal FA intake. CpG-606 methylation was higher in females than males. FA supplementation of pregnant dams increased CpG-508 methylation in females, but not males. Methylation of CpG-404 was increased in offspring of FS dams irrespective of sex. These findings show that maternal FA supplementation induced altered methylation of specific CpGs in the PEPCK promoter, which are located within or proximal to transcription factor-binding sites ${ }^{(4)}$. One implication of these findings is that increased maternal FA intake during pregnancy induced changes in the epigenetic regulation of genes, which may be cryptic unless the tissue is exposed to an appropriate environment challenge. If this was to occur in human subjects, these findings would support detailed investigation of the long-term consequences of current dietary recommendations to women for FA intake before and during pregnancy.

This work was supported by the Biotechnology and Biological Sciences Research Council. MAH receives salary support from the British Heart Foundation.

1. MRC Vitamin Study Group (1991) Lancet 338, 131-137.

2. Lillycrop KA, Rodford J, Garratt ES et al. (2010) Br J Nutr 103, 1711-1719.

3. Lillycrop KA, Phillips ES, Torrens C et al. (2008) Br J Nutr 100, 278-282.

4. Yang J, Reshef L, Cassuto H et al. (2009) J Biol Chem 284, 27031-27035. 